\title{
General synthesis of single atom electrocatalysts via a facile condensation-carbonization process
}

Received 00th January 20xx, Accepted 00th January 20xx DOI: $10.1039 / \times 0 \times x 00000 x$

\author{
Weiming Chen, ${ }^{\mathrm{a}}$ Xuanli Luo, ${ }^{\mathrm{c}}$ Thomas J A Slater, ${ }^{\mathrm{d}}$ Yongfang Zhou, ${ }^{\mathrm{a}}$ Sanliang Ling, ${ }^{\mathrm{c}}$ Rui Bao, ${ }^{\mathrm{e}}$ Jesum \\ Alves Fernandes, ${ }^{f}$ Jianshe Wang ${ }^{g}$ and Yi Shen*ab
}

\begin{abstract}
The general and cost-effective synthesis of single atom electrocatalysts (SAECs) still remains a great challenge. Herein, we report a general synthetic protocol for the synthesis of SAECs via a simple condensation-carbonization process, in which furfural and cyanamide were condensation polymerized in the presence of polystyrene nanospheres and metal ions, followed by a pyrolysis to N-doped carbon nanosheets (NCNSs) supported SAECs. Six types of SAECs containing platinum, palladium, gold, nickel, cobalt and iron were synthesized to demonstrate the generality of the synthesis protocol. This methodology affords a facile solution to the trade-off between support conductivity and metal loading of SAECs by optimizing the ratio of carbon/nitrogen precursors, i.e., furfural furfuryl and cyanamide. The presence of single metal atoms was confirmed by high-angle annular dark field scanning transmission electron microscopy and X-ray absorption fine structure measurements. The three-dimensional distribution of single platinum atoms was vividly revealed by depth profile analysis in the scanning transmission electron microscope. The resulting SAECs showed excellent performance for glycerol electro-oxidation and water splitting in alkaline solutions. Notably, Pt/NCNs possessed an unprecedent mass-normalized current density of $5.3 \mathrm{~A}$ per milligram of platinum, which is 32 times that of the commercial Pt/C catalyst. Density functional theory calculations were conducted to reveal the adsorption behavior of glycerol over the SAECs. Using Ni/NCNSs and Co/NCNSs as anodic and cathodic electrocatalysts, we constructed a solar panel powered electrolytic cell for overall water splitting, leading to an overall energy efficiency of $8.8 \%$, which has been among one of the largest solar-to-hydrogen conversion efficiencies reported in the literature.
\end{abstract}

\section{Introduction}

Electrocatalysis has played a central role in energy conversion and storage, and chemical synthesis. ${ }^{1,2}$ Searching for high-performance electrocatalysts has drawn tremendous attention from the research community in the last decade. ${ }^{3-5}$ Recently, single atom electrocatalysts (SAECS) have been of particular interest because of their maximum atomic utilization and unique electronic and geometric configurations. ${ }^{6,7}$ So far, many SAECs based on nickel, ${ }^{8}$ cobalt, ${ }^{9}$ iron, ${ }^{10}$ copper $^{11}$ zinc $^{12}{ }^{12}$ platinum, ${ }^{13}$ gold $^{2}$ and ruthenium ${ }^{14}$ have been reported for electrochemical processes such as the hydrogen evolution reaction, ${ }^{15}$ oxygen evolution reaction, ${ }^{16}$ oxygen reduction reaction, ${ }^{5} \mathrm{CO}_{2}$ reduction reaction ${ }^{17}$ and nitrogen reduction

\footnotetext{
a. School of Food Science and Engineering, South China University of Technology, Guangzhou 510641, China.Email: feyshen@scut.edu.cn (Dr Y. Shen).

b. Overseas Expertise Introduction Center for Discipline Innovation of Food Nutrition and Human Health (111 Center), Guangzhou, 510641, China.

c. Advanced Materials Research Group, Faculty of Engineering, University of

Nottingham, Nottingham, NG7 2RD, UK

d. Electron Physical Sciences Imaging Centre, Diamond Light Source Ltd., Oxfordshire OX11 ODE, UK.

e. Faculty of Materials Science and Engineering, Kunming University of Science and

Technology, Kunming 650093, China.

f. School of Chemistry, University of Nottingham, Nottingham, UK.

g. School of Chemical Engineering and Energy, Zhengzhou University, Zhengzhou 450001, China.

+ Electronic Supplementary Information (ESI) available: See DOI: 10.1039/ $\mathrm{x} 0 \mathrm{xx00000 \textrm {x }}$
}

reaction. ${ }^{18}$ These reported SAECs generally displayed superior catalytic performance relative to their counterparts (nanoparticles or clusters) because of a greater number of active sites per mass and prominent size effects, which give rise to distinctive adsorption and desorption behaviors of reactants/intermediates, thereby leading to enhanced activity and selectivity. ${ }^{19-21}$

Owing to the prominent merits, many studies have been devoted to developing efficient methods for the preparation of SAECs. ${ }^{22,23}$ To date, many synthetic strategies have been reported to fabricate SAECs such as atomic layer deposition, ${ }^{24}$ mass-selected softlanding, ${ }^{25}$ ball milling, ${ }^{26}$ coprecipitation, ${ }^{27}$ defect/vacancy confinement, $^{28}$ high-temperature pyrolysis, ${ }^{29}$ wetness impregnation, ${ }^{30}$ iced-photochemical reduction ${ }^{31}$ and ultra-lowtemperature synthesis ${ }^{32}$. Among the reported methods, pyrolysis of metal-organic-frameworks (MOFs) represents the most feasible approach for the production of SAECs. ${ }^{33-35}$ The SAECs resulting from the pyrolysis of MOFs show outstanding catalytic performance owing to the strong coordination interaction of single atoms with the nitrogen-doped carbon matrix. ${ }^{36,37}$ Unfortunately, the synthesis of MOFs can be complicated and costly, involving many expensive and toxic moieties, and therefore remains an obstacle for scalable production..$^{38}$ In addition, the choices of metal type and ligands are limited, rendering it challenging to fabricate SAECs bearing varying metal atoms. ${ }^{23,38}$ Furthermore, being an ideal electrocatalyst, it should possess excellent conductivity to facilitate the transport of electrons. On the other hand, the presence of abundant nitrogen 
atoms in the catalyst supports is indispensable to stabilize the single metal atoms, which could inevitably compromise the conductivity of the supports. ${ }^{20}$ This trade-off between catalyst conductivity and metal loading represents another obstacle for the synthesis of SAECs. Therefore, despite great progress on SAEC synthesis, a general synthesis protocol is highly desirable, but unfortunately still lacking. More recently, exciting results have been reported by several groups. ${ }^{39-42}$ Serendipitously, the authors found that the condensation product of furfural and cyanamide offers a general matrix for stabilizing single atoms. The prominent advantages of using furfural and cyanamide as chelating agents of metal ions lies in their low-cost and low-toxicity. The solvent for the condensation reaction is water, which renders the synthetic process highly sustainable. In addition, this synthetic protocol affords a facile solution to address the trade-off between catalyst conductivity and metal loading by optimizing the ratio of cyanamide/furfural. Six types of SAECs containing platinum, palladium, gold, nickel, cobalt and iron supported by nanoporous nitrogen doped carbon nanosheets
(M/NCNSs, M=Pt, Pd, Au, Ni, Co, Fe) were synthesized to demonstrate the generality of the synthesis protocol. The distribution of the single metal atoms in the catalyst was revealed via scanning transmission electron microscopy, including threedimensional reconstruction via a focal-series. The resulting SAECs showed excellent performance for glycerol electro-oxidation and water splitting. Notably, Pt/NCNSs possessed a unprecedent massnormalized current density of 5.3 A per milligram of platinum for glycerol electro-oxidation, which is 32 times that of the commercial $\mathrm{Pt} / \mathrm{C}$ catalyst. To explore the reason of such outstanding activity, density functional theory (DFT) calculations were conducted. $\mathrm{Ni} /$ NCNSs exhibited better activity toward the oxygen evolution reaction (OER), while Co/NCNSs exhibited superior activity toward the hydrogen evolution reaction (HER). Using Ni/NCNSs and Co/NCNSs as anodic and cathodic electrocatalysts, we constructed a solar panel powered electrolytic cell for overall water splitting, leading to a remarkable overall energy efficiency of $8.8 \%$.

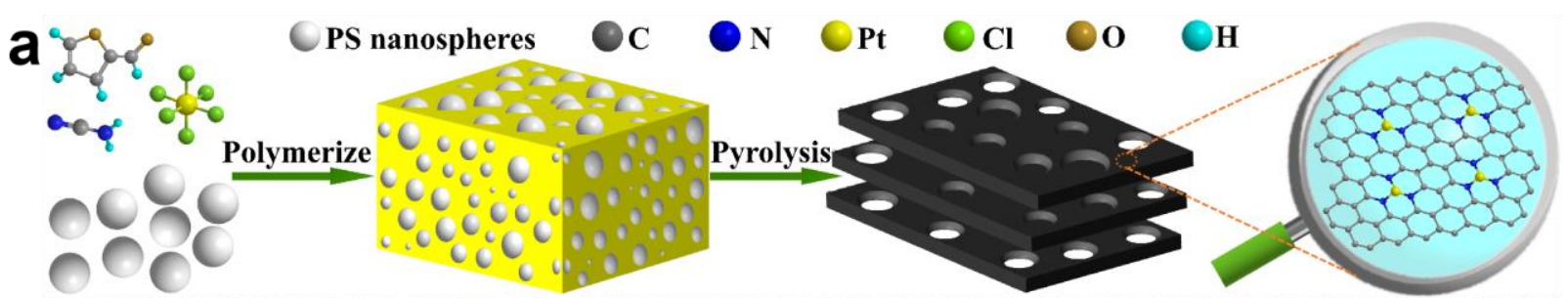

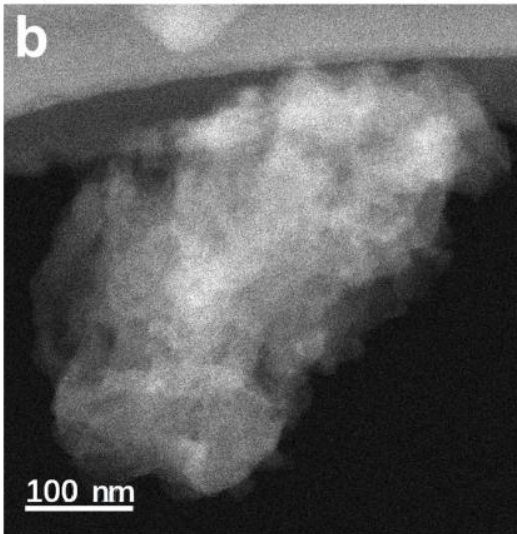

e

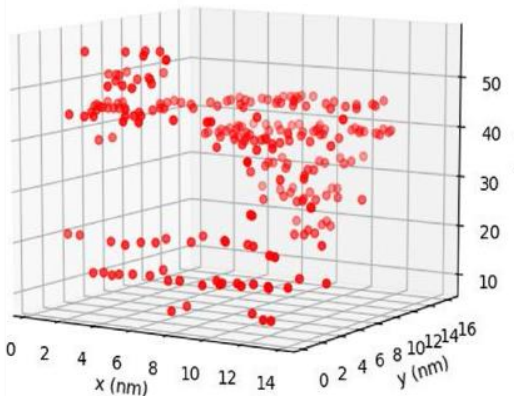

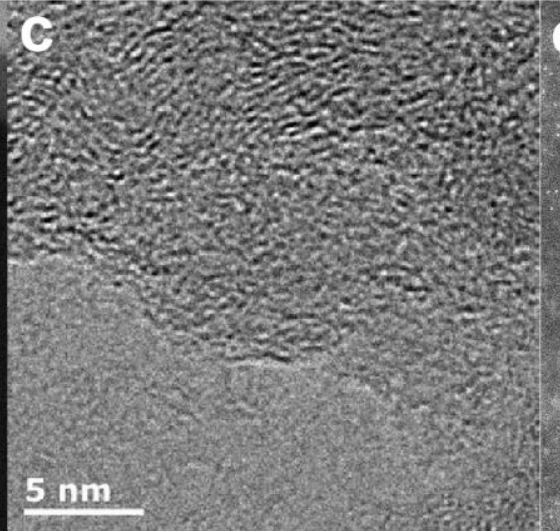

f

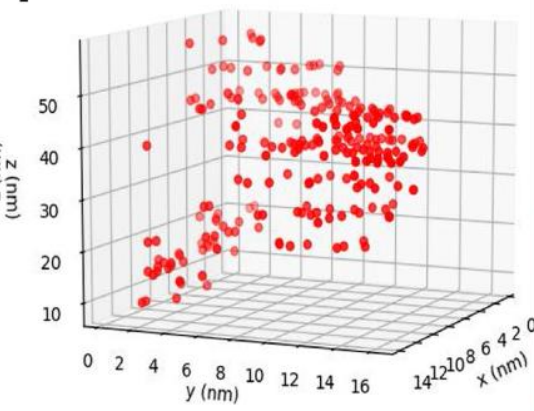

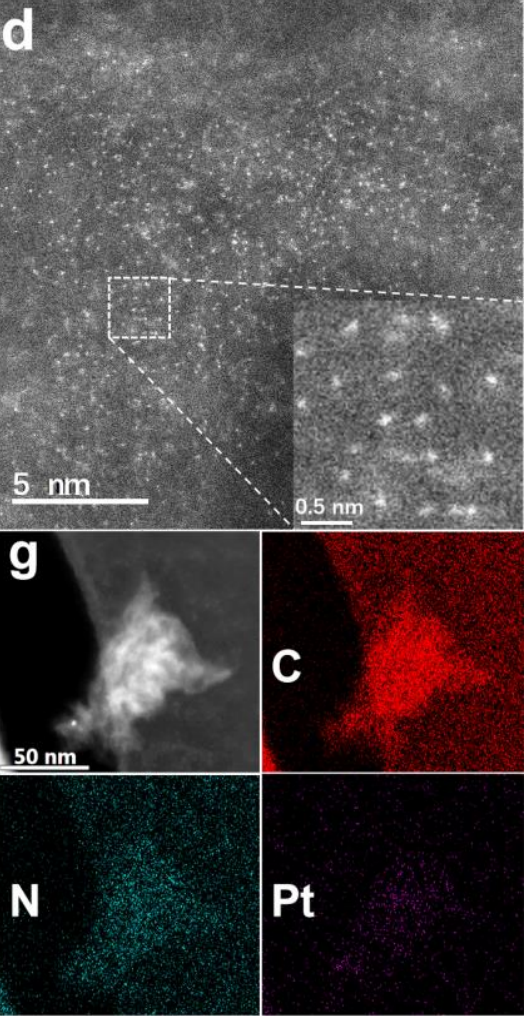

Fig. 1 Structural characterization of Pt/NCNSs. (a) schematic illustration of the synthetic process, (b) HAADF-STEM image, (c) high-resolution TEM image, (d) high-resolution HAADF-STEM image, inset of enlarged area, (e) and (f) three-dimensional distribution of platinum atoms reconstructed from a HAADF-STEM focal series, (g) HAADF-STEM image and corresponding elemental mapping of carbon, nitrogen and platinum, respectively. 


\section{Results and discussion}

The synthetic procedures are schematically shown in Fig. 1a. Furfural, which is employed as a typical carbon source, and cyanamide which is employed as a nitrogen source, were in-situ polymerized in the presence of PS nanospheres. The nitrogen and oxygen groups in the polymeric matrix serve as efficient chelating sites for metal cations, while the PS nanospheres were utilized as a sacrificial template to increase the surface area of the sample. After a simple pyrolysis process, the mixture was converted into single metal atoms supported by nitrogen-doped carbon nanosheets. Fourier-transform infrared spectroscopy analysis (Fig. S1a, ESI†) was conducted to reveal the changes in the surface groups of the samples. The FTIR profile of the polymer precursors are relatively complicated owing to the large number of groups in the sample. In contrast, after pyrolysis, only N-H $\left(3660 \mathrm{~cm}^{-1}\right), \mathrm{C}=\mathrm{C}(\mathrm{N})\left(1560 \mathrm{~cm}^{-1}\right)$, and $\mathrm{C}-\mathrm{C}(\mathrm{N})\left(1190 \mathrm{~cm}^{-1}\right)$ groups are observed in the samples, suggesting the transformation of the polymers into carbonaceous materials. ${ }^{43} \mathrm{Pt} / \mathrm{NCNSs}$ was first fabricated as a proof-of-concept example. Fig. $\mathbf{1 b}$ shows the overall view of the Pt/NCNSs sample in the form of a HAADF-STEM image. More TEM images of samples were shown in Fig. S1b-d (ESI+). The sample consists of aggregates of nanosheets. Notably, few nanoparticles can be found within the aggregates. High-resolution TEM images, as shown in Fig. 1c, confirmed the layered structure of the Pt/NCNSs sample. Fig. 1d shows a representative high-resolution HAADF-STEM image. It clearly reveals numerous single Pt atoms, as denoted by bright dots, which were highly dispersed on the nitrogen-doped carbon nanosheets. Since HAADF-STEM images only afford two-dimensional atom distribution, a focal series was performed on the Pt/NCNSs sample to reveal the three-dimensional dispersion of single metal atoms on the carbon matrix. The focal series allows reconstruction of the Pt atom locations as a function of depth through the sample. Pt atoms were observed throughout the support material, as indicated by the red dots in Fig. 1e and $\mathbf{f}$. Based on this depth profile analysis, the volume density of platinum atom is estimated to be 0.04 $\mathrm{Pt} \mathrm{nm}{ }^{-3}$, which is close to the theoretical value of $0.11 \mathrm{Pt} \mathrm{nm}^{-3}$. We note that while the focal steps were matched to the nominal depth of field and therefore Pt atoms should have been accurately counted, the volume density could vary by a factor of two. Corresponding elemental mapping, obtained through STEM-EDX (Fig. 1g), demonstrates that carbon, nitrogen and platinum are evenly distributed throughout one of the nanosheets.

The structure of the Pt/NCNSs was also studied by XRD, a representative spectrum of which is shown in Fig. S2a (ESIt). Compared with NCNSs, Pt/NCNSs exhibited two sharper diffraction peaks that can be related to graphitized carbon. It is noteworthy that no peaks associated with metallic platinum are observed from the XRD profiles. The structures of the samples were further characterized by Raman spectroscopy, as shown in Fig. S2b (ESI + ). Two typical peaks related to carbon materials including $D$ and $G$ bands are noted. The intensity ratio $\left(\mathrm{I}_{\mathrm{D}} / \mathrm{I}_{\mathrm{G}}\right)$ of Pt/NCNSs was determined to be 1.00, which is similar to that of NCNSs (1.03). Fig. S2c (ESIt) displays the $\mathrm{N}_{2}$ adsorption-desorption isotherm of Pt/NCNSs. It reveals a typical type IV isotherm with a distinct hysteresis loop, suggesting the presence of mesopores in the sample. Based on the isotherm, the Brunauer-Emmett-Teller (BET) surface area of Pt/NCNSs was calculated to be $552.4 \mathrm{~m}^{2} \mathrm{~g}^{-1}$. Such a large BET value is attributed to the thin-layered structure and presence of a number of mesopores. The pore size distribution of the Pt/NCNSs was also determined based on the adsorption branch of the isotherm, as shown in inset of Fig. S2c (ESIt). The average diameter of the pores was determined to be $4.8 \mathrm{~nm}$. It should be pointed out that the large surface aera and abundant mesopores are favorable for the dispersion and exposure of single Pt atoms. The electronic structures of Pt/NCNSs were investigated by XPS. Fig. S2d shows the survey spectrum. It reveals the presence of carbon, oxygen, nitrogen and platinum elements. No other elements are noted from the
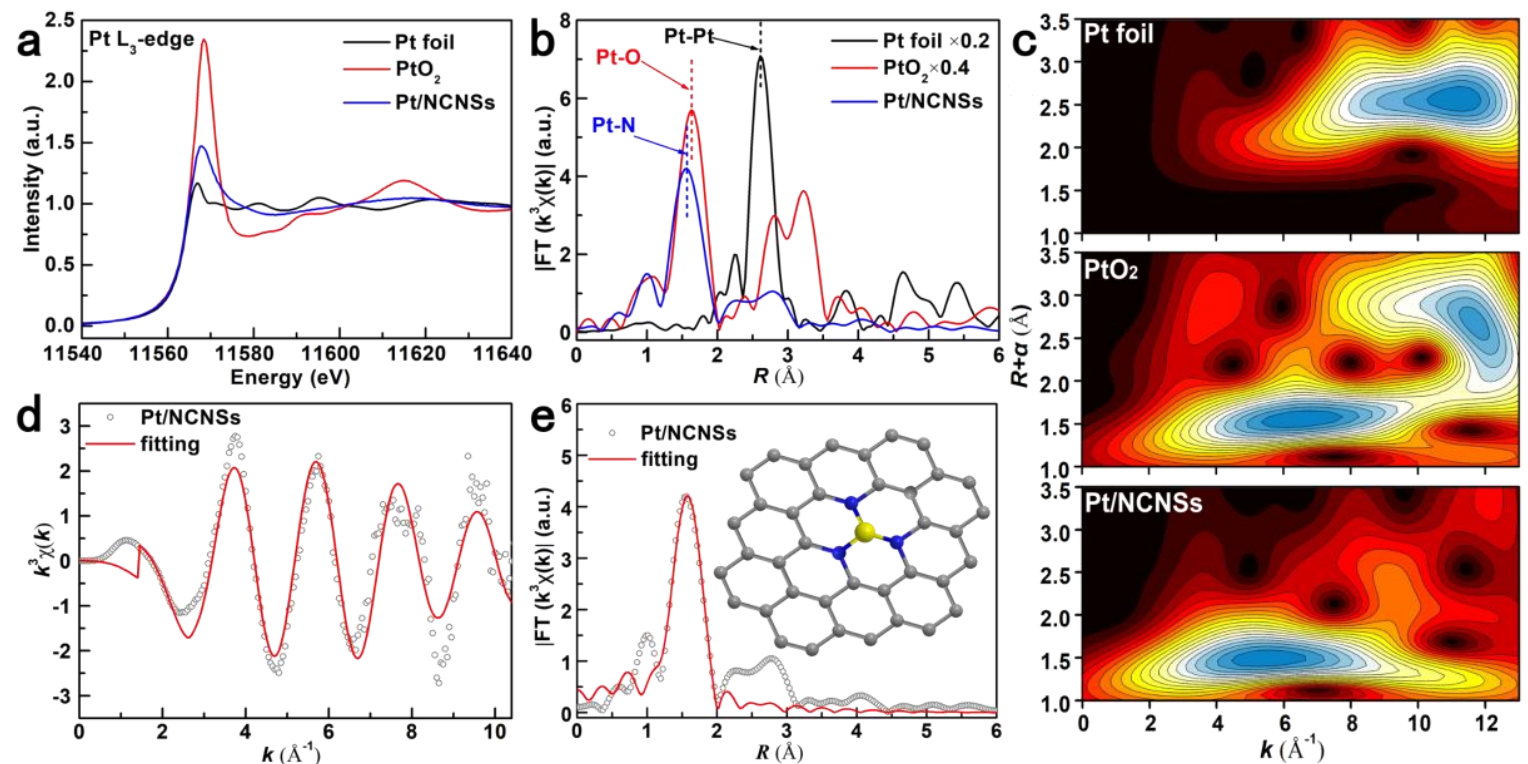

Fig. 2 Synchrotron XAFS measurement of Pt/NCNSs. (a) XANES spectra, (b) $k^{3}$-weighted FT-EXAFS of Pt/NCNSs and references at Pt L3 edge, (c) WT for the EXAFS signal of Pt/NCNSs and references, and $(d, e)$ Corresponding EXAFS fitting curves in $k$ and $R$ space, respectively. 
spectrum, indicating the high-purity of the sample. Fig. S2e and S2f $(E S I+)$ show the high-resolution $N$ 1s and $\mathrm{Pt} 4 \mathrm{f}$ spectra, respectively. The $N$ 1s spectrum was carefully decomposed. Five $N$ states including pyridinic- $\mathrm{N}, \mathrm{Pt}-\mathrm{N}_{\mathrm{x}}$, pyrrolic- $\mathrm{N}$, graphitic- $\mathrm{N}$ and oxidized-N were found, corresponding to binding energies of 397.8, 398.9, 399.9, 400.8, $402.0 \mathrm{eV}$, respectively. The Pt $4 \mathrm{f}$ spectrum consists of two peaks with binding energies of $75.4,72.1 \mathrm{eV}$, corresponding the doublets of $4 \mathrm{f}$ $5 / 2$ and $4 f 7 / 2$, respectively. Moreover, the binding energy of $P t 4 f$ spectrum are located between that of $\mathrm{Pt}^{0}$ and $\mathrm{Pt}^{2+}$, indicate that the valence state of platinum in Pt/NCNSs is $0^{\sim}+2$.

The chemical states and coordination environment of Pt species at the atomic level were further investigated by using element-selective $X$-ray absorption fine structure (XAFS) measurements. The normalized X-ray absorption near edge structure (XANES) spectra of the $\mathrm{Pt}_{3}$-edge for $\mathrm{Pt} / \mathrm{NCNSs}$ along with $\mathrm{Pt}$ foil and $\mathrm{PtO}_{2}$ references are shown in Fig. 2a. The white-line intensities in the normalized XANES spectra reflect the oxidation state of Pt species. ${ }^{44}$ It can be seen that the white-line intensities of Pt/NCNSs is higher than that of $\mathrm{Pt}$ foil but much lower than that of $\mathrm{PtO}_{2}$, indicating that the oxidation state of $\mathrm{Pt}$ in $\mathrm{Pt} / \mathrm{NCNSs}$ is between $\mathrm{Pt}^{0}$ and $\mathrm{Pt}^{4+}, 45$ which consistent with the results of XPS (Fig. S2f, ESI ${ }^{\dagger}$ ). The unique electronic structure of $\mathrm{Pt}$ species with positive charges is mainly due to the coordination of $\mathrm{Pt}$ with $\mathrm{N}$ atoms in the support which results in electron transfer from Pt to $\mathrm{N}$ atoms. ${ }^{46}$ Such an electron transfer could be also present in other SAECs reported in this study. The $\mathrm{k}^{3}$-weighted Fourier transform spectra in $R$ space of the $\mathrm{Pt}_{2}$-edge extended X-ray absorption fine structure (EXAFS) for Pt/NCNSs and references are shown in the Fig. 2b. Only one distinct peak (1.56 $⿱$ ) ), which is ascribed to the backscattering between Pt and light atoms can be observed at the EXAFS curve of Pt/NCNSs, which is shorter than that

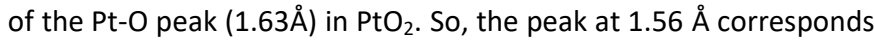
to the Pt-N/C first coordination shell in this system. No other appreciable peak can be observed in the $2.0 \sim 4.0 \AA$ interval, indicating that most of the Pt in Pt/NCNSs are atomically dispersed. In order to gain the elemental nature and radial distance resolution, wavelet transform (WT) analysis (Fig. 2c) in $k$ space of $\mathrm{Pt}^{\mathrm{L}_{3}}$ edge EXAFS was performed. The WT contour plot of Pt/NCNSs presents only one maximum intensity at $\sim 5.5 \AA^{-1}$, which is assigned to the Pt-N/C coordination. An intensity maximum at $\sim 11 \AA^{-1}$ associated with the Pt-Pt coordination is not detected, and the shape of intensity maximum for Pt/NCNSs is different from that of Pt-O coordination in $\mathrm{PtO}_{2}$. These suggest that no obvious $\mathrm{Pt}$ nanoparticles or $\mathrm{PtO}_{2}$ is present in Pt/NCNSs. Quantitative EXAFS curve fitting (Fig. 2d, e) was performed to reveal the coordination structure of $\mathrm{Pt}$ species in Pt/NCNSs. The fitting results leads to a prominent peak at $1.56 \AA$ which can be assigned to the Pt- $\mathrm{N}$ first shell coordination. The average coordination number for Pt-N species was estimated to be 3.0, and the average bond distance of Pt-N is $2.0 \AA$. Based on the experimental spectral results and EXAFS curve fitting, it can be concluded that each single Pt atom in Pt/NCNSs is coordinated to three nitrogen atoms and that the resulting $\mathrm{Pt}-\mathrm{N}_{3}$ species is stabilized by the nitrogen doped carbon matrix. The structure of the $\mathrm{Pt}-\mathrm{N}_{3}$ site is shown in the inset of Fig. 2 e.

The synthesis protocol was extended to fabricate other single-metal catalysts by varying metal precursors. Fig. 3 shows HAADF-STEM images of Pt/NCNSs, Au/NCNSs, Pd/NCNSs, Ni/NCNSs, Co/NCNSs, and Fe/NCNSs. It can be seen that similar to single Pt/NCNSs, single $\mathrm{Au}, \mathrm{Pd}, \mathrm{Ni}, \mathrm{Co}$ and Fe atoms supported by NCNSs are also successfully prepared by the synthetic procedures. The structures of the resulting single-atom catalysts were also characterized. Fig. S3 (ESI†) shows the XRD patterns of the single-atom catalysts. Notably, in contrast with other samples, the XRD profile of the Pd/NCNSs exhibited several small peaks, which can be assigned to metallic Pd nanoparticles. The Raman spectra of the samples shown in Fig. S4 (ESI + ) display typical peaks of carbon materials, confirming the formation of the nitrogen-doped carbon nanosheet support in the samples. The nitrogen adsorption-desorption isotherms of the samples are shown in Fig.s S5-S10 (ESI†). Similar to the Pt/NCNSs, all
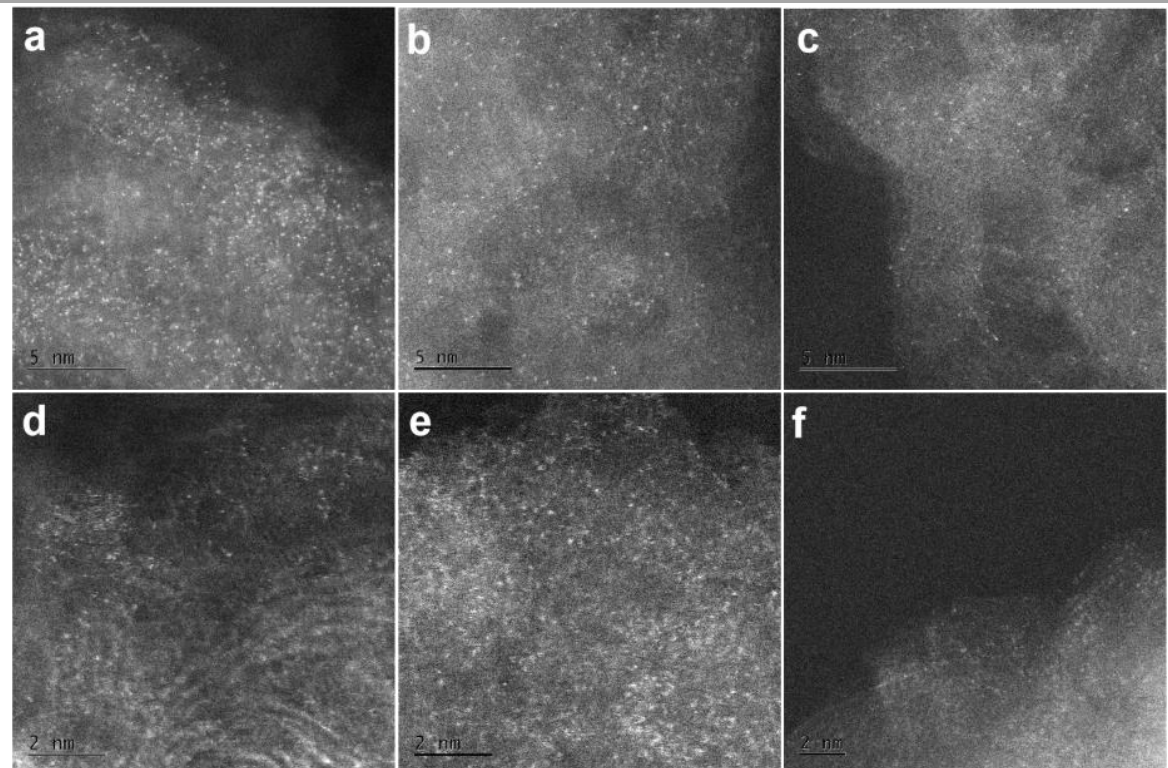

Fig. 3 Morphological structures of the SACs. High-resolution HAADF-STEM images of (a) Pt/NCNSs, (b) Au/NCNSs, (c) Pd/NCNSs, (d) Ni/NCNSs, (e) Co/NCNSs, and (f) Fe/NCNSs. 
the samples possess a Type IV isotherm with average pore sizes of 4$6 \mathrm{~nm}$. To investigate the effects of pyrolysis temperature in the physiochemical properties of the samples, the isotherms of Ni/NCNSs-800 and Ni/NCNSs-1000 are also shown in Fig. S11 and 12 (ESI + ), respectively. It was found that the Ni/NCNSs exhibited the largest BET surface area. The XPS spectra of the Au/NCNSs, $\mathrm{Pd} / \mathrm{NCNSs}, \mathrm{Co} / \mathrm{NCNSs}$, Fe/NCNSs and Ni/NCNSs are shown in Fig. S13, S14, S15, S16 and S17 (ESI+), respectively. It can be seen that the oxidation state of metal in samples were higher than corresponding foils. The unique electronic structure of metal species with positive charges is mainly due to the coordination of metal and support. The corresponding analysis results are summarized in Table S1 (ESI + ). To gain the exact loading of metal in samples, inductively coupled plasma optical emission spectrometer (ICP-AES) was performed. It can be seen that the metal loadings determined from the XPS results are consistent with those determined from ICP-AES results (Table S2, $\mathrm{ESI}+$ ). Since the nitrogen species play a critical role in stabilizing the single atoms, the $\mathrm{N} 1 \mathrm{~s}$ spectra were thoroughly analyzed. The results are included in Table $\mathbf{S 3}(\mathrm{ESI}+)$ ). Fig. $\mathbf{S 1 8}(\mathrm{ESI}+)$ shows the $\mathrm{N} 1$ s spectra of $\mathrm{Ni} / \mathrm{NCNSs}$ prepared at $800,900,1000$ and $1100^{\circ} \mathrm{C}$. The total $\mathrm{N}$ content decreases with increasing pyrolysis temperature. To further verify the formation of single atoms in the samples, XAFS measurements were conducted, as shown in Fig. S19-21 (ESI†). The corresponding fitting results are listed in Table S4 (ESIt). Interestingly, in the Co/NCNSs, Fe/NCNSs and Ni/NCNSs samples, each metal atom was coordinated by four nitrogen atoms. In contrast, in the Pt/NCNSs and Pd/NCNSs, each precious metal atom was surrounded by three nitrogen atoms.

The resulting Pt/NCNSs, Pd/NCNSs, and Au/NCNSs catalysts were employed as electrocatalysts for glycerol oxidation in alkaline solutions. Glycerol electro-oxidation is of great significance in terms of application to direct glycerol fuel cells. In addition, electrooxidation provides a totally green and efficient approach for the conversion of glycerol into value-added products.

For comparison, the single atom catalysts were benchmarked to corresponding commercial catalysts based on nanoparticles. Fig. 4a comparatively shows the $\mathrm{CV}$ curves of glycerol electro-oxidation over $\mathrm{Pt} / \mathrm{C}$ and Pt/NCNSs catalysts. The mass-normalized current density of

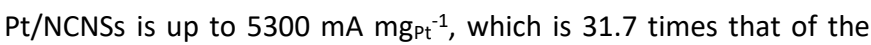
commercial Pt/C catalyst (167 mA mg $\left.{ }_{P_{t}}^{-1}\right)$. Shown in Fig. 4b, the current density of Au/NCNSs ( $3640 \mathrm{~mA} \mathrm{mg}^{-1}$ ) is 271 times that of $\mathrm{Au}$ nanoparticles. In contrast, the $\mathrm{Pd} / \mathrm{NCNSs}$ possessed a peak current density of $729.3 \mathrm{~mA} \mathrm{mg}^{-1}$, which is only 2.6 times that of a $\mathrm{Pd} / \mathrm{C}$
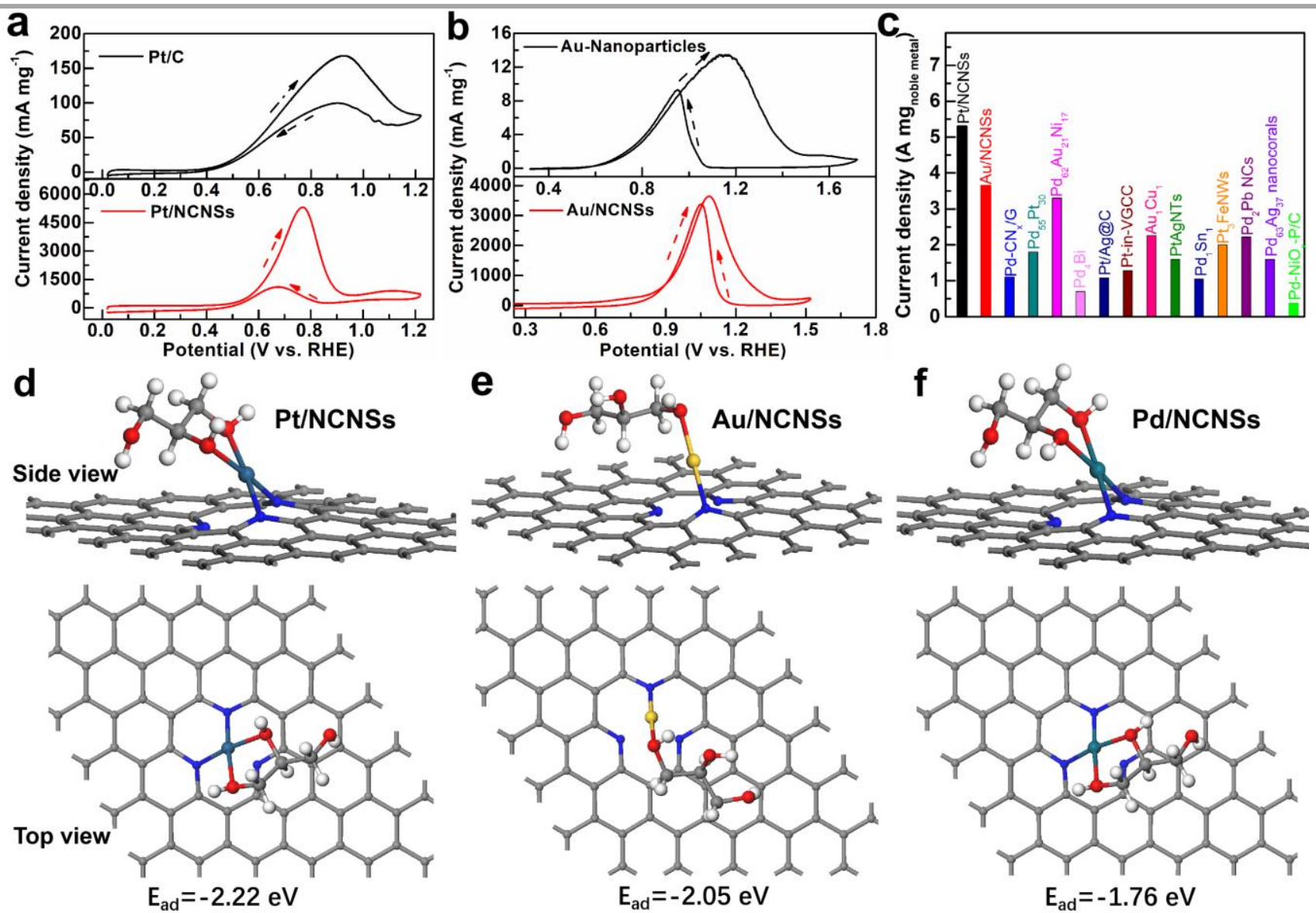

Fig. 4 Electrochemical performance of the samples for glycerol oxidation and water splitting. CV curves of glycerol electro-oxidation over (a) Pt/C and Pt/NCNSs and (b) Au nanoparticles and Au/NCNSs, and (c) comparison of mass activity of the electrocatalysts toward glycerol oxidation. Adsorption configurations and calculated adsorption energy ( $\left.E_{a d}\right)$ of glycerol molecules on single (d) Pt, (e) Au and (f) Pd atom catalysts. Color code: hydrogen - white, carbon - grey, oxygen - red, nitrogen - blue, gold - yellow, platinum/palladium - navy blue. 
catalyst (Fig. S22, ESI+). This could be attributed to the presence of Pd nanoparticles in the Pd/NCNSs, as suggested by evidence from the XRD and EXAFS results. To further highlight the performance of the single atom catalysts, the current densities of Pt/NCNSs and $\mathrm{Au} / \mathrm{NCNSs}$ were compared with those of electrocatalysts reported in the literature, as shown in Fig. $\mathbf{4 c}$ and Table S5 (ESI + ). It can be seen that the Pt/NCNSs and Au/NCNSs outperformed all reported electrocatalysts, rendering them as the most active catalysts for glycerol electro-oxidation. To explore the origin of excellent catalytic activity, DFT calculations (see Support Information for computational details) were performed. Fig. $\mathbf{4 d}$-f show the optimized adsorption configurations of glycerol molecules on the single Pt, Au and Pd atom electrocatalysts. Two additional adsorption configurations of glycerol on the Au/NCNSs were considered, see optimized geometries in Fig. S23 (ESI + ), and it turns out both configurations are less stable than the configuration shown in Fig. 4e. Therefore, we did not explore other adsorption configurations of glycerol molecules on single $\mathrm{Pt} / \mathrm{Pd}$ atom electrocatalysts. The adsorption energies are shown in Fig. 4d-f. The adsorption energy of glycerol on Pt/NCNs was calculated to be $-2.22 \mathrm{eV}$, which is larger than those on Au/NCNSs ($2.05 \mathrm{eV}$ ) and Pd/NCNSs (-1.76 eV). Note that the adsorption energy is well correlated with the catalytic activity, indicating that the adsorption of glycerol is the critical step for glycerol electrooxidation. Further inspection reveals that the adsorption energy of glycerol on the SAEC is larger than that on the corresponding nanoparticles. For instance, the adsorption energy of glycerol on $\mathrm{Pt}(111), \mathrm{Pt}(110)$ and $\mathrm{Pt}(100)$ were reported (using the same DFT method that was used in the current study) to be $-1.29,-1.71$ and $1.31 \mathrm{eV}$, respectively, ${ }^{47}$ all of which are lower than that on the Pt/NCNSs. In addition, the adsorption energy of glycerol on the $\mathrm{Pd} / \mathrm{NCNSs}(-1.76 \mathrm{eV})$ is also larger than that on Pd(111), i.e.-1.21 eV, as reported in a previous computational study using the same DFT method. ${ }^{48}$ These results manifest that the outstanding catalytic activity of the SAECS is possibly attributed to the atomic distribution as well as their unique electronic structures.

The effects of pyrolysis temperature and metal loading on the catalytic performance of $\mathrm{Au} / \mathrm{NCNSs}$ were also investigated. Fig. S24a $(E S I+)$ shows the CV curves of glycerol oxidation over the Au/NCNSs samples obtained from varying pyrolysis temperatures. It reveals that the Au-NCNS-900 had a larger peak current density than AuNCNS-800 and Au-NCNS-850. The loading of Au also affects the catalytic performance, as shown in Fig. S24b (ESIt). The Au-NCNS with an Au weight percentage of $0.4 \%$ exhibited the largest peak current density. These findings indicate that the pyrolysis temperature and metal loading affected the formation of single atoms. The stability of the catalysts was investigated as shown in Fig. S25 (ESI $)$. The current density of Au/NCNSs show negligible decline after 500 cycles, indicate the excellent cyclic performance.

Electrochemical water splitting represents a sustainable approach for hydrogen production. Developing high-performance and costeffective HER and OER electrocatalysts is of paramount importance for the widespread application of water splitting techniques. SAECS based on nickel, cobalt and iron were promising candidates for OER and HER catalysts by rational design. ${ }^{23}$ The general synthetic protocol afforded a facile solution to the trade-off between N/C ratio (support conductivity) and metal loading of SAECs by optimizing the ratio of furfural and cyanamide, which provides an avenue to improve catalytic performance. To ensure that metal ions were converted into single atoms successfully, pyrolysis temperature was optimized first. As shown in Fig. S26 $(\mathrm{ESI}+$ ), the single nickel atom catalyst showed the best OER activity with a pyrolysis temperature of $900^{\circ} \mathrm{C}$ . Then, the effects of the volumetric ratio of cyanamide and furfural in the feedstock, in addition to metal loading, on OER activity of the single nickel atom catalyst were investigated, as shown in Fig. S27 and S28 (ESI + ). It was found that the single nickel atom catalyst prepared with a cyanamide/furfural ratio of 1:1 and a metal loading (metal in feedstock) of $0.5 \mathrm{wt} \%$ (Ni/NCNSs) showed the best OER activity. By optimizing the preparation conditions, the Ni/NCNSs showed excellent OER activity, better than the $\mathrm{IrO}_{2}$ catalyst that is regarded as a benchmark for OER.

In addition to the Ni-based catalyst, SAECs based on cobalt and iron were also synthesized at optimized preparation conditions. The resulting single transition metal atom catalysts were studied as both HER and OER electrocatalysts. Fig. 5a shows the OER polarization curves. For comparison, the results of NCNSs and a precious $\mathrm{IrO}_{2}$ catalyst are also included. It indicates that the Ni/NCNSs shows the highest current density. The Co/NCNSs possessed lower current density at low overpotentials but outperformed the $\mathrm{IrO}_{2}$ at high overpotentials. Specifically, as shown in Fig. $\mathbf{5 c}$, to yield a current density of $10 \mathrm{mV} \mathrm{cm}^{-2}$, the overpotentials of the samples follow the sequence of $\mathrm{Ni} / \mathrm{NCNSs}(297 \mathrm{mV})<\mathrm{IrO}_{2}(322 \mathrm{mV})<\mathrm{Co} / \mathrm{NCNSs}(345$ $\mathrm{mV}$ ) < Fe/NCNSs $(429 \mathrm{mV})<\mathrm{NCNSs}(466 \mathrm{mV})$. In contrast, to obtain a current density of $50 \mathrm{mV} \mathrm{cm}^{-2}$, the sequence changes to be Ni/NCNSs $(335 \mathrm{mV})<\mathrm{Co} / \mathrm{NCNSs}(392 \mathrm{mV})<1 \mathrm{rO}_{2}(404 \mathrm{mV})<\mathrm{Fe} / \mathrm{NCNSs}(485 \mathrm{mV})$ $<$ NCNSs $(702 \mathrm{mV})$.

Fig. $\mathbf{5 d}$ shows the Tafel slope of the catalysts for the OER. It reveals that the Ni/NCNSs exhibits a Tafel slope of $42.7 \mathrm{mV} \mathrm{dec}^{-1}$, which is smaller than those of $\mathrm{IrO}_{2}\left(45.6 \mathrm{mV} \mathrm{dec}^{-1}\right)$, Co/NCNSs $(62.7 \mathrm{mV} \mathrm{dec}$ 1), Co-NCNSs (75.3 mV dec ${ }^{-1}$ ) and NCNSs (134.6 mV dec $\left.{ }^{-1}\right)$. Fig. $\mathbf{S 2 9}$ (ESI + ) shows the TOF values of the catalysts. Compared to the $\mathrm{IrO}_{2}$, the single atom catalysts possessed much larger TOF values. In particular, the Ni/NCNSs exhibited the best intrinsic OER activity. To further highlight the OER activity of the single atom catalysts, the performance of $\mathrm{Ni} / \mathrm{NCNSs}$ and $\mathrm{Co} / \mathrm{NCNSs}$ are compared with those of electrocatalysts reported in the literature, shown in Table S6 (ESI + ). This clearly reveals that the Ni/NCNSs and Co/NCNSs prepared here are among the best reported OER electrocatalysts so far.

The stability of the single atom catalysts was investigated by chronoamperometric measurements, as shown in Fig. S30 and S31 $(E S I+)$. It indicates that all the catalysts are highly stable for the OER. To recover the metal distribution of single atoms after stability test, high-resolution HAADF-STEM tests were performed on the spent samples. Fig. $\mathbf{S 3 2}(\mathrm{ESI}+)^{)}$shown the HAADF-STEM images of fresh and spent $\mathrm{Co} / \mathrm{NCNSs}$. Isolated cobalt atoms are anchoring on carbon matrix uniformly before the chronoamperometric measurements (Fig. 3e and Fig. S32a, ESI $)$ ). However, some raft-like cobalt clusters are observed after a $12 \mathrm{~h}$ chronoamperometric measurements (Fig. S32d-f, ESI + ), indicating that migration and aggregation occurred during the test. Although single cobalt atoms were migrated, the current density of Co/NCNSs shown limited decline during the testing time (Fig. S32b, ESI†). This mainly due to that there are still a large 

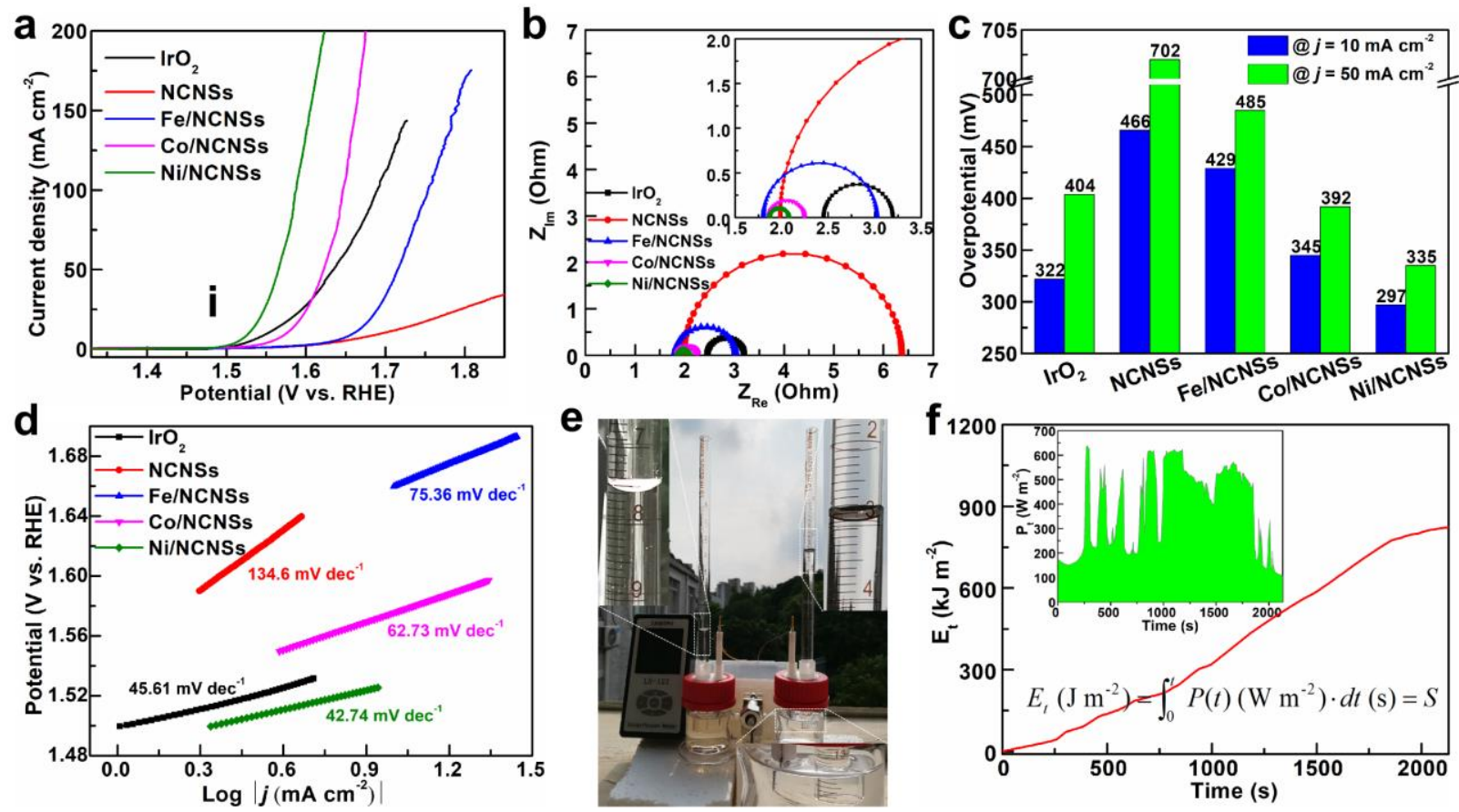

Fig. 5 Electrochemical performance of the samples for water splitting. (a) OER polarization curves recorded in a solution of $1 \mathrm{M}$ KOH with a scan rate of $2 \mathrm{mV}$ $\mathrm{s}^{-1}$, (b) EIS spectra recorded at an applied potential of $200 \mathrm{mV}$, (c) comparison on overpotentials at OER current densities of 10 and $50 \mathrm{~mA} \mathrm{~cm}^{-2}$, (d) Tafel slopes of the catalysts for OER, (e) digital photos of a solar panel powered set-up after electrolysis for 2130 seconds, (f) energy density of the sunlight during the electrolysis process, inset shows the power density of sunlight in real time.

number of single atoms anchoring on the support (Fig. S32g, h, ESI + ), and that raft-like cobalt clusters are highly exposed. Expectedly, the spent Au/NCNSs shows similar characteristics to the spent Co/NCNSs (Fig. S33, ESIt). To explore the reason for the superior activity of the $\mathrm{Ni} / \mathrm{NCNSs}$, ESI measurements were conducted, as shown in Fig. $\mathbf{5 b}$. Based on the Nyquist plots, the values of series resistance $\left(R_{s}\right)$ and charge transfer resistance $\left(R_{c t}\right)$ were determined. All three single atom and pure NCNSs catalysts exhibited smaller $R_{s}$ values compared with that of $\mathrm{IrO}_{2}$. This could be attributed to the better electronic conductivity of the carbonaceous materials compared to the oxide. The Ni/NCNSs and Co/NCNSs possessed $R_{c t}$ values of 0.21 and $0.38 \Omega$, respectively, which are smaller than those of $\mathrm{IrO}_{2}(0.74 \Omega)$, $\mathrm{Fe} / \mathrm{NCNSs}$ $(1.2 \Omega)$ and NCNSs $(4.4 \Omega)$, indicting faster kinetics of the OER over the $\mathrm{Ni} / \mathrm{NCNS}$ and Co/NCNSs, which is consistent with the polarization results.

In addition, since the electrochemically active surface area is closely related to the double-layer capacitance $\left(C_{\text {dl }}\right)$ of the catalyst, cyclic voltammetry curves were recorded in a potential range of $0.15 \sim 0.25$ $V$ with scan rates of $10,20,40,60,80$ and $100 \mathrm{mV} \mathrm{s}^{-1}$ to determine the $C_{d l}$ values of the catalyst, as shown in Fig. S34 (ESI ${ }^{\dagger}$ ). A good linear relationship between the scan rate and $\left(j_{a}-j_{c}\right) / 2$ was obtained, as shown in Fig. $\mathbf{S 3 5}$ (ESIt), and the $C_{d l}$ value was determined by the slope of the curve accordingly. The Co/NCNSs, Ni/NCNSs, Fe/NCNSs and NCNSs had $C_{d l}$ values of 14.4, 11.5, 4.7 and $10.2 \mathrm{mF} \mathrm{cm}^{-2}$ respectively, which cannot be well correlated with the HER or OER activity of the samples. This suggests that the intrinsic activity of M$\mathrm{N}_{\mathrm{x}}$ moieties is highly dependent on the metal atoms.

The HER performance of the single atom catalysts was also evaluated and further benchmarked against the precious Pt/C. Fig. S36 (ESI+) shows the HER polarization curves. It shows that the current densities of the single atom catalysts are much larger than that of NCNSs, but lower than that of the Pt/C catalysts. Specifically, as shown in Fig. S37 (ESIt), to obtain a current density of $10 \mathrm{~mA} \mathrm{~cm}{ }^{-2}$, the $\mathrm{Ni} / \mathrm{NCNS}$, Co/NCNSs and Fe/NCNSs require overpotentials of 258, 221 and $311 \mathrm{mV}$, respectively, which are lower than that of NCNSs $(415 \mathrm{mV})$, but higher than that of Pt/C (53 mV). Based on the polarization curves, the Tafel slopes of the catalysts are determined, as shown in Fig. $\mathbf{S 3 8}$ (ESI+). The Ni/NCNSs, Co/NCNSs and Fe/NCNSs had Tafel slopes of 162,134 and $240 \mathrm{mV} \mathrm{dec}^{-1}$, which is smaller than that of the NCNCs $\left(425 \mathrm{mV} \mathrm{dec}{ }^{-1}\right.$ ), but larger than that of the Pt/C catalyst $\left(35.1 \mathrm{mV} \mathrm{dec}^{-1}\right)$. To reveal the intrinsic HER activity, TOF values of the samples are calculated, as shown in Fig. S39 (ESI†). At lower overpotentials, the transition metal single atom catalysts possessed lower TOF values compared with those of the Pt/C. However, with increasing overpotential, the TOF values of the transition metal single atom catalysts outnumbered those of the $\mathrm{Pt} / \mathrm{C}$. Notably, among the three single atom catalysts, the Co/NCNSs exhibited the best HER activity.

Inspired by the remarkable performance of the single-atom catalysts, the Ni/NCNSs and Co/NCNSs were utilized as anodic and cathodic electrocatalysts for overall water splitting. Continuous hydrogen and oxygen bubbles were generated from the electrolysis cell powered by a $2.0 \mathrm{~V}$ solar panel (see video). The digital photos of the experimental setup before and after the electrolysis process are shown in Fig. 5e and S40 (ESI $)$ ), respectively. After $2130 \mathrm{~s}, 5.2 \mathrm{~mL}$ of oxygen and $10.1 \mathrm{~mL}$ of hydrogen were obtained from the cathode and anode, respectively. The volume ratio of oxygen to hydrogen is close to the theoretical value of 1:2. To calculate the overall energy efficiency of solar-to-hydrogen, the power density of the sunlight 
was recorded by a power meter as shown in the inset of Fig. $\mathbf{5 f}$, and the energy density was determined accordingly as shown in Fig. $\mathbf{5 f}$. The overall energy efficiency of the solar-hydrogen transformation process was calculated to be $8.8 \%$. Such a solar-to-hydrogen energy efficiency was among one of the largest values reported in the literature as shown in Table S7 (ESIt).

\section{Conclusions}

In summary, a versatile condensation-carbonization synthetic methodology was reported for the synthesis of a series of SAECs containing platinum, palladium, gold, nickel, cobalt and iron. Furfural and cyanamide were used as both ligands to stabilize metal ions and carbon/nitrogen sources while polystyrene nanospheres were utilized as a template to increase the surface area. This method could well address the trade-off between $\mathrm{N} / \mathrm{C}$ ratio (support conductivity) and metal loading by optimizing the ratio of furfural and cyanamide. HAADF-STEM and XAFS measurements unambiguously confirmed the presence of abundant single atoms supported by NCNSs. Depth profile analysis using a HAADF-STEM focal series was performed on $\mathrm{Pt} / \mathrm{NCNSs}$ to uncover the three-dimensional distribution of single platinum atoms. The resulting Pt/NCNSs, Pd/NCNSs and Au/NCNSs were examined as electrocatalysts for glycerol electro-oxidation while Ni/NCNSs, Co/NCNSs and Fe/NCNSs were studied as electrocatalysts for the HER and OER. The Pt/NCNSs possessed the largest current density of $5.3 \mathrm{~A}$ per milligram of platinum, which is 32 times that of commercial Pt/C and is larger than any value we could find in the literature. The Co/NCNSs exhibited superior HER activity while $\mathrm{Ni} / \mathrm{NCNSs}$ had superior OER activity. Using Ni/NCNSs and Co/NCNSs as anodic and cathodic electrocatalysts, we constructed a $2.0 \mathrm{~V}$ solar panel powered electrolytic cell for overall water splitting, leading to a remarkable overall energy efficiency of $8.8 \%$. The synthetic protocol reported in this work could pave a new route to synthesize high-performance SAECs.

\section{Experimental}

\section{Synthesis of polystyrene nanospheres}

Polystyrene nanospheres were synthesized via a method reported in the authors' previous work. ${ }^{49}$

\section{Synthesis of M/NCNSs (M=Ni, Co, Fe, Pt, Au, and Pd)}

Typically, $8 \mathrm{~mL}$ of PS suspension (16 wt.\%), $2 \mathrm{~mL}$ of cyanamide solution, $2 \mathrm{~mL}$ of furfural, $0.4 \mathrm{~mL}$ of $0.1 \mathrm{M} \mathrm{HCl}$ solution and a predetermined volume of metal solution with a concentration of 10 $\mathrm{g} \mathrm{L}^{-1}$ of were introduced into a sealed $50 \mathrm{~mL}$ tube. The metal precursors were $\mathrm{NiCl}_{2} \cdot 6 \mathrm{H}_{2} \mathrm{O}, \mathrm{CoCl}_{2} \cdot 6 \mathrm{H}_{2} \mathrm{O}, \mathrm{FeCl}_{3} \cdot 6 \mathrm{H}_{2} \mathrm{O}, \mathrm{H}_{2} \mathrm{PtCl}_{6} \cdot 6 \mathrm{H}_{2} \mathrm{O}$, $\mathrm{HAuCl}_{4} \cdot 3 \mathrm{H}_{2} \mathrm{O}$ and $\mathrm{PdCl}_{2}$ for the synthesis of Ni/NCNSs, Co/NCNSs, $\mathrm{Fe} / \mathrm{NCNSs}, \mathrm{Pt} / \mathrm{NCNSs}, \mathrm{Au} / \mathrm{NCNSs}$ and $\mathrm{Pd} / \mathrm{NCNSs}$, respectively. The mixture was magnetically stirred at $60^{\circ} \mathrm{C}$ for $36 \mathrm{~h}$. Afterwards, the stopper of the tube was removed and the solvent in the mixture, that is, water, was slowly evaporated in $48 \mathrm{~h}$. Subsequently, the mixture was first dried at $100^{\circ} \mathrm{C}$ for $48 \mathrm{~h}$ using an oven and then transferred into a tube furnace and pyrolyzed in a nitrogen atmosphere. The furnace was heated to a given temperature with a heating rate of 4 ${ }^{\circ} \mathrm{C} \mathrm{min}^{-1}$, held at temperature for $3 \mathrm{~h}$, and then cooled to ambient temperature with a rate of $6{ }^{\circ} \mathrm{C} \mathrm{min}^{-1}$. The resulting powder was ground by agate mortar. For convenience, the resulting samples were denoted as M/NCNSs-T, where $M$ is the metal type and T is the pyrolysis temperature. For comparison, nitrogen doped carbon nanosheets free of any metal were also prepared.

\section{Structural characterization}

The morphology of the samples was studied by field emission scanning electron microscopy (FESEM) (JSM-7600F, JEOL) and transmission electron microscopy (JEM2010, JEOL). To observe the single atoms in the samples, high-angle annular dark field STEM images were obtained from an alternative microscope (ARM200F JEOL) at the electron Physical Sciences Imaging Centre (ePSIC) at Diamond Light Source. An acceleration voltage of $200 \mathrm{kV}$ was used, with a convergence semi-angle of $23 \mathrm{mrad}$ and an ADF inner collection angle of $67 \mathrm{mrad}$. The beam current was $13 \mathrm{pA}$. For the focal series, a convergence semi-angle of $32 \mathrm{mrad}$ was used, to give a depth of field of approximately $3 \mathrm{~nm}$. A focal step of $3 \mathrm{~nm}$ was used to match the step to the nominal depth of field. An energy dispersive $\mathrm{X}$-ray (EDX) analyzer equipped in the TEM and an axis-ultra X-ray photoelectron spectrometer (Kratos-Axis Ultra System) with

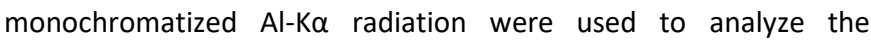
elemental composition of the samples. The X-ray absorption fine structure spectra were recorded from beamline B18 at Diamond Synchrotron Irradiation Facility with stored electron energy of 2.2 $\mathrm{GeV}$ using transmission mode. The spectra were processed following the conventional procedure using the IFEFFIT package. X-ray diffraction (XRD) patterns were obtained by a diffractometer (PW1830, Philips) equipped with Cu-Ka radiation of $1.54 \AA$ A. Raman spectra were recorded with a LabRAM Aramis microscope using 532$\mathrm{nm}$ excitation at room temperature. The nitrogen isotherms were recorded using a WBS-820 analyzer. FTIR measurements were performed using a VERTEX 33 spectroscopy.

\section{Electrochemical measurements}

Electrochemical measurements were performed on an electrochemical station (CHI 660E) connecting with a typical threeelectrode cell. Catalyst powder was dispersed into a mixture of distilled water, ethanol, isopropanol, ethylene glycol and Nafion solution (5 wt.\%) with a volumetric ratio of 1000:750:150:95:5 by ultrasonication at least 30 minutes. The weight percentage of the catalyst powder in the resulting suspension was $1.6 \mathrm{mg} \mathrm{mL}^{-1}$. For water splitting, $80 \mathrm{uL}$ of the suspension was deposited onto a carbon paper with a geometric area of $10 \times 10 \mathrm{~mm}$. For glycerol electrooxidation, $10 \mathrm{uL}$ of the suspension was deposited onto a glassycarbon electrode with a diameter of $3 \mathrm{~mm}$. These electrodes were thoroughly dried before the electrochemical measurements. A carbon rod and $\mathrm{Hg} / \mathrm{HgO}$ electrode were used as counter and reference electrodes, respectively. The electrolyte solutions are $1 \mathrm{M}$ $\mathrm{KOH}$ and $0.5 \mathrm{M} \mathrm{KOH}+0.5 \mathrm{M} \mathrm{GLY}$ for the OER/HER and glycerol electrooxidation reaction, respectively. The scanning rate are $2 \mathrm{mV} \mathrm{s}^{-1}$ and $50 \mathrm{mV} \mathrm{s}^{-1}$ for the LSV curve of OER/HER and CV curve of glycerol electro-oxidation reaction, respectively.

\section{Computational details}

All density functional theory (DFT) calculations, including structural optimizations and total energy calculations, were performed using 
the Vienna Ab initio Simulation Package ${ }^{50}$ with periodic boundary conditions. We used a plane-wave basis set with a kinetic energy cutoff of $400 \mathrm{eV}$ to expand the wave functions. The Perdew-BurkeErnzerhof functional ${ }^{51}$ in combination with the projector augmented wave method ${ }^{52,53}$ were used to solve the Kohn-Sham equations. The Grimme's D3 van der Waals correction ${ }^{54}$ with the Becke-Johnson damping 55 was included to account for possible dispersion interactions between glycerol molecule and our single atom electrocatalysts. A first-order Methfessel-Paxton smearing ${ }^{56}$ with a width of $0.2 \mathrm{eV}$ was used to improve the convergence of electronic self-consistent field calculations. All DFT calculations were spinunpolarized, and have been performed in the $\Gamma$-point approximation for sufficiently large cells; we used a $6 \times 6$ supercell of single layer graphene, with a vacuum slab of $\sim 15 \AA$ thickness. An energy convergence threshold of $10^{-4} \mathrm{eV}$ was used for all total energy calculations, and the structural optimizations, including cell parameters and atomic positions, were considered converged if all interatomic forces fall below $0.01 \mathrm{eV} / \mathrm{A}$. The Bader's charge analysis was performed using the method developed by Henkelman et al. ${ }^{57}$ Following the EXAFS curve fitting (see Fig. 2 and Table S4, ESI + ), which demonstrates that both Pt and Pd atoms are coordinated to three nitrogen atoms, we considered a structural model where a single $\mathrm{Pd} / \mathrm{Pt} / \mathrm{Au}$ metal atom is stabilized by three pyridinic nitrogen atoms (see Fig. 2e). The optimised structure of glycerol molecule adsorbed on supported single $\mathrm{Pd} / \mathrm{Pt} / \mathrm{Au}$ atom is shown in Fig. $4 \mathrm{~d}-\mathrm{f}$. For $\mathrm{Au}$, two additional configurations (see Fig. S23, ESI ${ }^{\dagger}$ ) were considered, which are higher in energy than the minimum energy structure (see Fig. 4e) by 11.1 and $13.4 \mathrm{~kJ} / \mathrm{mol}$ respectively. These two configurations will not be discussed further.

\section{Conflicts of interest}

There are no conflicts to declare.

\section{Acknowledgements}

The project was financially supported by the National Natural Science Foundation of China (Grant No. 21706081) and 111 Project (B17018). The authors thank Diamond Light Source for the support from the electron Physical Science Imaging Centre for the use of Instrument E01 under proposal number MG23723. The authors thank Professors Alan Chadwick and Giannantonio Cibin for the XAS measurements, and the Diamond Light Source for provision of beam time through the Block Allocation Group (BAG) for Energy Materials under proposal sp17198. Dr Luo acknowledges the support of a Daphne Jackson Trust fellowship and its sponsors are Royal Academy of Engineering and the University of Nottingham. We acknowledge the use of Athena at HPC Midlands+, which was funded by the EPSRC on grant EP/P020232/1, as part of the HPC Midlands+ consortium. We are also grateful for access to the University of Nottingham's Augusta HPC service.

\section{Notes and references}

1. Z. W. Seh, J. Kibsgaard, C. F. Dickens, I. Chorkendorff, J. K. Norskov and T. F. Jaramillo, Science, 2017, 355, 6321.

2. S. Cao, M. Yang, A. O. Elnabawy, A. Trimpalis, S. Li, C. Wang, F.
Goltl, Z. Chen, J. Liu, J. Shan, M. Li, T. Haas, K. W. Chapman, S. Lee, L. F. Allard, M. Mavrikakis and M. Flytzani-Stephanopoulos, Nat. Chem., 2019, 11, 1098-1105.

3. X. Su, X. F. Yang, Y. Huang, B. Liu and T. Zhang, Acc. Chem. Res., 2019, 52, 656-664.

4. Y. Zhao, T. Ling, S. Chen, B. Jin, A. Vasileff, Y. Jiao, L. Song, J. Luo and S. Z. Qiao, Angew. Chem. Int. Ed., 2019, 58, 12252-12257.

5. D. Iglesias, A. Giuliani, M. Melchionna, S. Marchesan, A. Criado, L. Nasi, M. Bevilacqua, C. Tavagnacco, F. Vizza, M. Prato and P. Fornasiero, Chem, 2018, 4, 106-123.

6. Y. Chen, S. Ji, C. Chen, Q. Peng, D. Wang and Y. Li, Joule, 2018, 2, 1242-1264.

7. B. Zhang, G. Sun, S. Ding, H. Asakura, J. Zhang, P. Sautet and N. Yan, J. Am. Chem. Soc., 2019, 141, 8185-8197.

8. H. B. Yang, S.-F. Hung, S. Liu, K. Yuan, S. Miao, L. Zhang, X. Huang, H.-Y. Wang, W. Cai, R. Chen, J. Gao, X. Yang, W. Chen, Y. Huang, H. M. Chen, C. M. Li, T. Zhang and B. Liu, Nat. Energy, 2018, 3, 140147.

9. Z. Du, X. Chen, W. Hu, C. Chuang, S. Xie, A. Hu, W. Yan, X. Kong, X. Wu, H. Ji and L. J. Wan, J. Am. Chem. Soc., 2019, 141, 3977-3985.

10. H. Zhang, J. Li, S. Xi, Y. Du, X. Hai, J. Wang, H. Xu, G. Wu, J. Zhang, J. Lu and J. Wang, Angew. Chem. Int. Ed., 2019, 58, 14871-14876.

11. W. Li, C. Min, F. Tan, Z. Li, B. Zhang, R. Si, M. Xu, W. Liu, L. Zhou, Q. Wei, Y. Zhang and X. Yang, ACS Nano, 2019, 13, 3177-3187.

12. J. Li, S. Chen, N. Yang, M. Deng, S. Ibraheem, J. Deng, J. Li, L. Li and Z. Wei, Angew. Chem. Int. Ed., 2019, 58, 7035-7039.

13. L. DeRita, J. Resasco, S. Dai, A. Boubnov, H. V. Thang, A. S. Hoffman, I. Ro, G. W. Graham, S. R. Bare, G. Pacchioni, X. Pan and P. Christopher, Nat. Mater., 2019, 18, 746-751.

14. W. H. Lai, L. F. Zhang, W. B. Hua, S. Indris, Z. C. Yan, Z. Hu, B. Zhang, Y. Liu, L. Wang, M. Liu, R. Liu, Y. X. Wang, J. Z. Wang, Z. Hu, H. K. Liu, S. L. Chou and S. X. Dou, Angew. Chem. Int. Ed., 2019, 58, 11868-11873.

15. S. Ye, F. Luo, Q. Zhang, P. Zhang, T. Xu, Q. Wang, D. He, L. Guo, Y. Zhang, C. He, X. Ouyang, M. Gu, J. Liu and X. Sun, Energy Environ. Sci., 2019, 12, 1000-1007.

16. J. Zhang, Y. Zhao, X. Guo, C. Chen, C.-L. Dong, R.-S. Liu, C.-P. Han, Y. Li, Y. Gogotsi and G. Wang, Nat. Catal., 2018, 1, 985-992.

17. K. Jiang, S. Siahrostami, A. J. Akey, Y. Li, Z. Lu, J. Lattimer, Y. Hu, C. Stokes, M. Gangishetty, G. Chen, Y. Zhou, W. Hill, W.-B. Cai, D. Bell, K. Chan, J. K. Nørskov, Y. Cui and H. Wang, Chem, 2017, 3, 950960.

18. Y. Wang, X. Cui, J. Zhao, G. Jia, L. Gu, Q. Zhang, L. Meng, Z. Shi, L. Zheng, C. Wang, Z. Zhang and W. Zheng, ACS Catal., 2018, 9, 336344.

19. M. Liu, L. Wang, K. Zhao, S. Shi, Q. Shao, L. Zhang, X. Sun, Y. Zhao and J. Zhang, Energy Environ. Sci., 2019, 12, 2890-2923.

20. Y. Wang, J. Mao, X. Meng, L. Yu, D. Deng and X. Bao, Chem. Rev., 2019, 119, 1806-1854.

21. Q. Wang, Y. Lei, D. Wang and Y. Li, Energy Environ. Sci., 2019, 12, 1730-1750.

22. L. Zhang, K. Doyle-Davis and X. Sun, Energy Environ. Sci., 2019, 12, 492-517.

23. H. Fei, J. Dong, D. Chen, T. Hu, X. Duan, I. Shakir, Y. Huang and X. Duan, Chem. Soc. Rev., 2019, 48, 5207-5241.

24. H. Yan, Y. Lin, H. Wu, W. Zhang, Z. Sun, H. Cheng, W. Liu, C. Wang, J. Li, X. Huang, T. Yao, J. Yang, S. Wei and J. Lu, Nat. Commun., 2017, 8, 1070.

25. S. Abbet, A. Sanchez, U. Heiz and W.-D. Schneider, J. Catal., 2001, 198, 122-127.

26. X. Cui, H. Li, Y. Wang, Y. Hu, L. Hua, H. Li, X. Han, Q. Liu, F. Yang, L. He, X. Chen, Q. Li, J. Xiao, D. Deng and X. Bao, Chem, 2018, 4, 1902-1910.

27. B. Qiao, A. Wang, X. Yang, L. F. Allard, Z. Jiang, Y. Cui, J. Liu, J. Li and T. Zhang, Nat. Chem., 2011, 3, 634-641.

28. L. Zhang, Y. Jia, G. Gao, X. Yan, N. Chen, J. Chen, M. T. Soo, B. Wood, D. Yang, A. Du and X. Yao, Chem, 2018, 4, 285-297.

29. H. Zhang, P. An, W. Zhou, B. Y. Guan, P. Zhang, J. Dong and X. W. 
D. Lou, Sci. Adv., 2018, 4, 6657.

30. Q. Shen, C. Cao, R. Huang, L. Zhu, X. Zhou, Q. Zhang, L. Gu and W. Song, Angew. Chem. Int. Ed., 2020, 59, 1216-1219.

31. H. Wei, K. Huang, D. Wang, R. Zhang, B. Ge, J. Ma, B. Wen, S. Zhang, Q. Li, M. Lei, C. Zhang, J. Irawan, L.-M. Liu and H. Wu, Nat. Commun., 2017, 8.

32. K. Huang, L. Zhang, T. Xu, H. Wei, R. Zhang, X. Zhang, B. Ge, M. Lei, J. Y. Ma, L. M. Liu and H. Wu, Nat. Commun., 2019, 10, 606

33. C.-C. Hou, H.-F. Wang, C. Li and Q. Xu, Energy Environ. Sci., 2020, DOI: 10.1039/c9ee04040d.

34. Q. Zuo, T. Liu, C. Chen, Y. Ji, X. Gong, Y. Mai and Y. Zhou, Angew. Chem. Int. Ed., 2019, 58, 10198-10203.

35. Y. Pan, C. Zhang, Z. Liu, C. Chen and Y. Li, Matter, 2020, 2, 78-110.

36. J. Wu, L. Xiong, B. Zhao, M. Liu and L. Huang, Small Methods, 2019, 4, 1900540.

37. T. He, S. Chen, B. Ni, Y. Gong, Z. Wu, L. Song, L. Gu, W. Hu and X. Wang, Angew. Chem. Int. Ed., 2018, 57, 3493-3498.

38. Y. S. Wei, M. Zhang, R. Zou and Q. Xu, Chem. Rev., 2020, DOI: 10.1021/acs.chemrev.9b00757.

39. H. Yang, L. Shang, Q. Zhang, R. Shi, G. I. N. Waterhouse, L. Gu and T. Zhang, Nat. Commun., 2019, 10, 4585.

40. X. He, Q. He, Y. Deng, M. Peng, H. Chen, Y. Zhang, S. Yao, M. Zhang, D. Xiao, D. Ma, B. Ge and H. Ji, Nat. Commun., 2019, 10, 3663.

41. L. Zhao, Y. Zhang, L. B. Huang, X. Z. Liu, Q. H. Zhang, C. He, Z. Y. Wu, L. J. Zhang, J. Wu, W. Yang, L. Gu, J. S. Hu and L. J. Wan, Nat. Commun., 2019, 10, 1278.

42. G. Zhang, Y. Jia, C. Zhang, X. Xiong, K. Sun, R. Chen, W. Chen, Y. Kuang, L. Zheng, H. Tang, W. Liu, J. Liu, X. Sun, W.-F. Lin and H. Dai, Energy Environ. Sci., 2019, 12, 1317-1325.

43. Y. Xiong, J. Dong, Z. Q. Huang, P. Xin, W. Chen, Y. Wang, Z. Li, Z. Jin, W. Xing, Z. Zhuang, J. Ye, X. Wei, R. Cao, L. Gu, S. Sun, L. Zhuang, X. Chen, H. Yang, C. Chen, Q. Peng, C. R. Chang, D. Wang and Y. Li, Nat. Nanotechnol., 2020, 15, 390-397.

44. K. Jorissen and J. Rehr, Journal of Physics: Conference Series, 2013, 430, 012001.

45. X. Li, X. Huang, S. Xi, S. Miao, J. Ding, W. Cai, S. Liu, X. Yang, H. Yang, J. Gao, J. Wang, Y. Huang, T. Zhang and B. Liu, J. Am. Chem. Soc., 2018, 140, 12469-12475.

46. Y. Zhu, W. Sun, J. Luo, W. Chen, T. Cao, L. Zheng, J. Dong, J. Zhang, M. Zhang, Y. Han, C. Chen, Q. Peng, D. Wang and Y. Li, Nat. Commun., 2018, 9, 3861.

47. P. Tereshchuk, A. S. Chaves and J. L. F. Da Silva, J. Phys. Chem. C, 2014, 118, 15251-15259.

48. P. C. D. Mendes, R. Costa-Amaral, J. F. Gomes and J. L. F. Da Silva, Phys. Chem. Chem. Phys., 2019, 21, 8434-8444.

49. B. Gong, Y. Peng, Z. Pan, W. Chen, Y. Shen, K. Xiao and L. Zhang, Chem. Commun., 2017, 53, 12766-12769.

50. G. Kresse and J. Furthmuller, Phys. Rev. B, 1996, 54, 11169-11186.

51. J. P. Perdew, K. Burke and M. Ernzerhof, Phys. Rev. Lett., 1996, 77, 3865-3868.

52. P. E. Blochl, Phys. Rev. B, 1994, 50, 17953-17979.

53. D. Hobbs, G. Kresse and Hafner, J. Phys. Rev. B, 2000, 62, 1155611570.

54. S. Grimme, J. Antony, S. Ehrlich and H. Krieg, J. Chem. Phys., 2010, 132, 154104.

55. S. Grimme, S. Ehrlich and L. J. Goerigk, Computat. Chem., 2011, 32, 1456-1465.

56. M. Methfessel and A. T. Paxton, Phys. Rev. B, 1989, 40, 3616-3621.

57. G. Henkelman, A. Arnaldsson and H. Jonsson, Computat. Mater. Sci., 2006, 36, 354-360. 